\title{
MANAGING GRB AFTERGLOWS OPTICAL/IR OBSERVATIONS IN THE WEB 2.0 ERA
}

\author{
D. Ricci ${ }^{1}$ and L. Nicastro ${ }^{1}$
}

\begin{abstract}
We present an overview of top internet technologies that can be used to build webtools and rich internet applications for astronomy. The aim is to simplify the data handling, reduction and access, in particular of optical/infrared images collected by traditional, automatic or robotic telescopes. These tools are particularly suitable for real-time management of GRB afterglow observations. Using these technologies we are developing a web-based images database management system. We present available features and discuss further improvements to the mentioned system.
\end{abstract}

\section{Introduction}

Modern web-based technologies are a unique opportunity for the astronomical community to simplify and modernize their work. A researcher is not simply a computer power user, but also, or "especially", an experienced internet user. For that reason, tasks like 1. browsing a database to seek information about catalogues, astronomical objects, images, etc., 2. scheduling/launching remote observations, 3 . performing simple statistics or data pre-reduction, 4. saving the analysis results for further purposes/analysis, all can be accomplished using nothing but an updated web browser.

We suggest to develop modern web-based tools as an alternative to old desktopbased instruments in particular to browse in a graphical and intuitive way data archives and analysis results (see e.g. Fig. 1). In fact, the management of a large amount of data produced every night by several ground- or space-based optical, infrared or other wavelength telescopes, is a primary problem in astronomy ${ }^{2}$.

${ }^{1}$ INAF/Istituto di Astrofisica Spaziale e Fisica Cosmica, Bologna, via Gobetti 101, 40129 Bologna, Italy

${ }^{2}$ Virtual Observatories efforts (www.ivoa.net/) are targeted at putting together and making accessible the astronomical data, both with stand-alone and webtools, and most of all at promoting common protocols and exchange formats. 


\section{User layer technologies}

A web page is an already accepted universal standard to interact with a computer. It hides to the end user all the low-level components that can evolve independently because of their modularity. Furthermore it is easily and generally available via any browser and is built on "generic" user's experience. Finally the possibility of customization are almost infinite. We suggest to build astronomical webtools by adopting the following new web technologies: html5 and css3 to boost the contents (W3C Schools 2011). In particular the new revision of the html language provides: the <canvas> element for raster graphic applications, the $\langle$ svg $>$ tag for inline vector graphics, the WebGL API for rich 3D applications, MathML and LATEX-like tags to simplify the presentation of scientific results, local storage and application cache to build offline webtools; d3.js, astro.js to build javascript dynamic plots and charts into an <svg $\rangle$ element (Bostock 2012) and to implement specific astronomical tools, as for example a fits file explorer in a <canvas > element; jquery to perform easy javascript client-side and server-side asynchronous requests (jQuery Foundation 2009). This library is simple, modular, widely used and easily extensible with the possibility to write plugins for the community.

\section{Management layer technologies}

We suggest the so-called LAMP platform for the content management. The concept is generic enough to be portable to other operating systems without any particular effort. This layer consists in the set up of a php class to interact with the web page (via jquery) and another that uses mysqli to communicate with the MySQL database server, or even call custom C++ APIs. The advantages of this approach consist in portability: by completely separating the server-side content, it is simpler to provide html templates that can be used to implement the same server functions in languages other than php; team development: the separation of the modules and the object-oriented approach are very convenient choices for the development of collaborative projects.

\section{Current development and further improvements}

We applied the concept mentioned in the previous sections to implement a webtool for the initialization, set-up and browsing of an images archive in a graphical and intuitive way. Currently our system is being developed within the GLORIA project. It supports the creation of a MySQL database for astronomical images management starting from user-selected images, e.g. collected by various telescopes. It allows to associate the fits header common keywords to a set of user-defined database fields by using custom metadata tables. A configurable php class provides the interaction with the database. After that, a MCS based C++ program (Nicastro \& Calderone 2007) is available to feed images into the DB (local or remote). We are extending the capabilities of this layer: association of user-chosen 

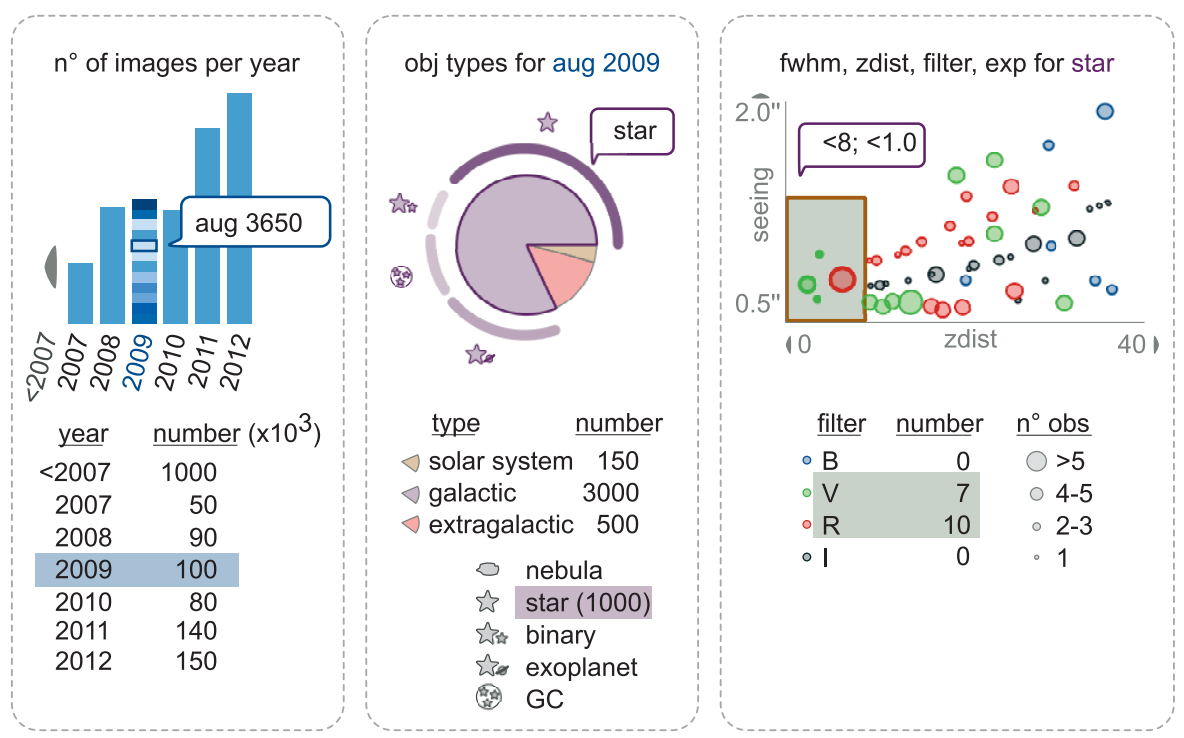

Fig. 1. Example of graphical browsing of a database of astronomical images.

keywords; definition of user accounts, access privileges and preferences (custom archive view). Our final goals are the following: graphical browsing of an image archive fed by an unlimited number of telescopes/cameras (see Fig. 1); access and use of object catalogs to perform visual and interactive analysis; interactive tools that mimic/extend ds9-saoimage (with dynamic cuts, statistics, etc.). The whole code will be released as free and open source software.

GLObal Robotic telescopes Intelligent Array for e-Science (GLORIA) is a project funded by the European Union Seventh Framework Programme (FP7/2007-2012) under grant agreement number 283783 .

\section{References}

Bostock, M., 2012, Data-Driven Documents, http://d3js.org/

jQuery Foundation, 2009, Jquery official website, http://jquery.com/

Nicastro, L., \& Calderone, G., 2007, Astron. Soc. Pacific Conf. Ser., Vol. 376, Astronomical Data Analysis Software and Systems XVI, ed. R.A. Shaw, F. Hill \& D.J. Bell, 323

W3C Schools, 2011, HTML5, CSS3, PHP, jQuery web references, http://www.w3schools.com/default.asp 
